\title{
BMJ Open Early low-energy versus high-energy enteral nutrition support in patients with traumatic intracerebral haemorrhage: protocol for a randomised controlled trial
}

\author{
Yanfei Shen, Xuping Cheng, Manzhen Ying, Weimin Zhang, Xuandong Jiang, \\ Kailei Du
}

To cite: Shen $\mathrm{Y}$, Cheng $\mathrm{X}$, Ying $M$, et al. Early lowenergy versus high-energy enteral nutrition support in patients with traumatic intracerebral haemorrhage: protocol for a randomised controlled trial. BMJ Open 2017;7:e019199. doi:10.1136/ bmjopen-2017-019199

- Prepublication history for this paper is available online. To view these files, please visit the journal online (http://dx.doi. org/10.1136/bmjopen-2017019199).

Received 17 August 2017 Revised 7 November 2017 Accepted 8 November 2017

CrossMark

Department of Intensive Care Unit, Dongyang People's Hospital, Jinhua, Zhejiang, China

Correspondence to

Dr Kailei Du;

dukailei1223@126.com

\section{ABSTRACT}

Background Early enteral nutrition (EN) is associated with shorter hospital stay and lower infection and mortality rates in patients with intracerebral haemorrhage. However, high-energy support always causes clinical complications, such as diarrhoea and aspiration pneumonia, and the true benefit of high-energy support in these patients has not been investigated. The appropriate amount of energy support still needs further investigation. Therefore, we are performing a randomised controlled trial to investigate whether early low-energy EN can decrease mortality and feeding-related complications and improve neurological outcomes as compared with high-energy EN in traumatic intracerebral haemorrhage (TICH) patients.

Methods/analysis This is a randomised, single-blind clinical trial performed in one teaching hospital. $220 \mathrm{TICH}$ patients will be randomly allocated to one of two groups in a 1:1 ratio: an intervention group, and a control group. The intervention group will receive early low-energy EN (10 kcal $/ \mathrm{kg} /$ day) and the control group will receive highenergy EN ( $25 \mathrm{kcal} / \mathrm{kg} /$ day) for 7 days. All these patients will be followed up for 90 days. The primary outcome is all-cause 90-day mortality. Secondary outcomes include the modified Rankin score, Glasgow Outcome Scale (GOS) and the National Institutes of Health Stroke Scale (NIHSS). Outcomes will be assessed at admission, 7, 30 and 90 days after onset of this trial. The safety of EN strategies will be assessed every day during hospitalisation.

Ethics and dissemination The trial will be conducted in accordance with the Declaration of Helsinki and has been approved by the ethics committee of Dongyang People's Hospital. The findings will be published in peer-reviewed medical journals.

Trial registration number ChiCTR-INR-17011384; Preresults.

\section{BACKGROUND}

Traumatic intracerebral haemorrhage (TICH) is a leading cause of longterm disability and mortality worldwide, resulting in great economic and healthcare burden. ${ }^{12}$ Nutritional support is of critical
Strengths and limitations of this study

- This is the first randomised, single-blind, controlled study investigating the appropriate amount of enteral nutrition for traumatic intracerebral haemorrhage patients.

- A preliminary trial including 70 patients was performed for crude efficacy evaluation and sample size calculation.

- Clinicians will not be blinded in this study, which may lead to potential bias.

importance because a hypermetabolic, hypercatabolic state is inevitable after brain insult. $^{3}$

Enteral feeding is preferred as it can stimulate gastrointestinal motility and prevent bacterial translocation. ${ }^{4}$ Seule $e t a \tilde{}{ }^{\tilde{}}$ suggested that infusion of enteral nutrition (EN) should begin as early as possible in TICH patients and was associated with clinical benefits such as alleviation of the hypercatabolic state, negative nitrogen balance and infections. ${ }^{67}$

However, the appropriate amount of EN for TICH patients remains unclear; many patients receiving high EN support have experienced feeding-related complications because delayed gastric emptying ${ }^{8}$ and decreased tone of the lower oesophageal sphincter $^{9}$ are common in neurologically injured patients. Efforts have been made to explore the balance between calorie intake and clinical outcomes in unspecified critically ill patients, although the conclusions were inconsistent.

A randomised trial performed in critically ill patients revealed that an underfeeding caloric intake strategy $(60-70 \%$ vs $90-100 \%$ of calculated target) was associated with lower mortality than target feeding. ${ }^{10}$ Similar 
findings were also reported in several cohort studies which showed that lower caloric intake was associated with significantly decreased hospital mortality, infection rate, mechanical ventilation duration and longer hospital stay, ${ }^{11}$ and may contribute to faster recovery. ${ }^{12}$ Yet, Petros et al reported a contrary finding that, compared with normal caloric feeding, hypocaloric feeding in the first 7 days was associated with more nosocomial infections but less gastrointestinal intolerance in critically ill patients. $^{13}$

Currently, one guideline on nutritional support suggests a low-energy enteral intake strategy during the critical course to maintain intestinal integrity and function while lowering complications. ${ }^{14}$ However, the heterogeneity in unspecified critically ill patients is non-negligible and may partly explain the inconsistent findings. ${ }^{1013}$

As no report has been made about the appropriate amount of EN for TICH patients, we conducted a randomised trial to investigate whether early low-energy EN could decrease mortality and feeding-related complication rates compared with early high-energy EN.

\section{METHODS}

\section{Study design}

This is a single-centre, randomised, single-blind clinical trial and will be carried out from October 2017 to October 2018. The study will be conducted in the intensive care unit (ICU) of Dongyang People's Hospital, a teaching hospital in China. Patients admitted to the ICU after neurological surgery due to TICH will undergo baseline evaluation before enrolment, including personal history records and laboratory tests, and informed consent will be signed by the direct relative of each participant before enrolment. Included patients will be randomised into two groups in a 1:1 ratio-a low-energy EN (10 kcal/ $\mathrm{kg} /$ day) group and a high-energy EN (25 kcal/ kg/day) group-and the intervention will last for 7 days. All these patients will be followed up for 90 days. All outcomes will be assessed at baseline, 7, 30 and 90 days after onset of the trial. The safety of the EN strategies will be assessed every day during hospitalisation. The trial is being conducted in accordance with the World Medical Association Declaration of Helsinki and has been approved by the ethics committee of Dongyang People's Hospital. The findings will be published in peer reviewed medical journals and presented at relevant conferences. The trial was registered at the Chinese Clinical Trial Registry (ChiCTR-INR-17011384) on 12 May 2017.

\section{Sample size}

The trial aims to detect if low-energy EN can decrease 90-day mortality in TICH patients, as compared with high-energy nutritional support. However, only a few studies have reported the association between the amount of calorie intake and clinical outcome in other diseases, such as acute respiratory distress syndrome (ARDS). ${ }^{15}$ Thus, we conducted a preliminary trial including 70
TICH patients (the randomisation and blinding method were not strictly followed) and we found that the 90-day mortality rates were $7.5 \%(3 / 40)$ in the low-energy group and $16.7 \%(5 / 30)$ in the high-energy group $(\mathrm{P}=0.233)$. With a power of $80 \%$ to detect a two-sided significance level of $5 \%, 194$ participants with complete data will be needed for this trial. With an estimated loss to follow-up rate of $10 \%, 220$ participants will therefore be required for the trial.

\section{Recruitment and inclusion criteria}

All patients with TICH admitted to the ICU of Dongyang People's Hospital will be screened for recruitment. Patients will be included if the following inclusion criteria are met: (1) older than 18 years; (2) admitted to hospital within 24 hours after injury, TICH diagnosed by brain CT, and the patient has undergone neurological surgery; (3) Glasgow Coma Score (GCS) before surgery was $\geq 5$ and $\leq 12$; (4) haemodynamics stable without any vasopressors; (V) informed consent obtained.

\section{Exclusion criteria}

Patients with one of the following will be excluded: (1) other types of intracerebral haemorrhage (ICH), such as aneurysmal subarachnoid haemorrhage and spontaneous ICH; (2) anticipated death within 2 days after ICU admission; (3) severe hepatic, renal or thyroid dysfunction or other metabolic diseases; (4) severe abdominal injury; (5) a history of stroke; (6) pregnant or breast-feeding; (7) not suitable according to a clinician's evaluation.

\section{Withdrawal criteria}

Patients will withdraw from this trial if one of the following criteria is met: (1) poor compliance; (2) EN intolerance leading to serious complications such as severe diarrhoea, vomiting and intra-abdominal hypertension; (3) other situations which require EN to be suspended for more than 48 hours. Participants may withdraw from this trial for any reason. EN will not be combined with other treatments in this trial.

\section{Randomisation and allocation concealment}

The randomisation code will be generated using SPSS software and kept in opaque sealed envelopes. The whole randomisation process will be performed by an independent clinician who is familiar with statistics. The sealed envelopes are preserved by this clinician until the end of the trial. Clinicians will divide the included patients into two groups using the random numbers printed on the envelopes which are assigned according to the patients' screening sequence numbers.

\section{Blinding}

This is a single-blind (patient-blinding) trial. The clinicians are unlikely to be blinded from the allocation as the amount of enteral intake will obviously be different in the two groups. However, the clinicians responsible for the outcome assessment and data analysis will be blinded from this allocation until the whole trial has been completed. 


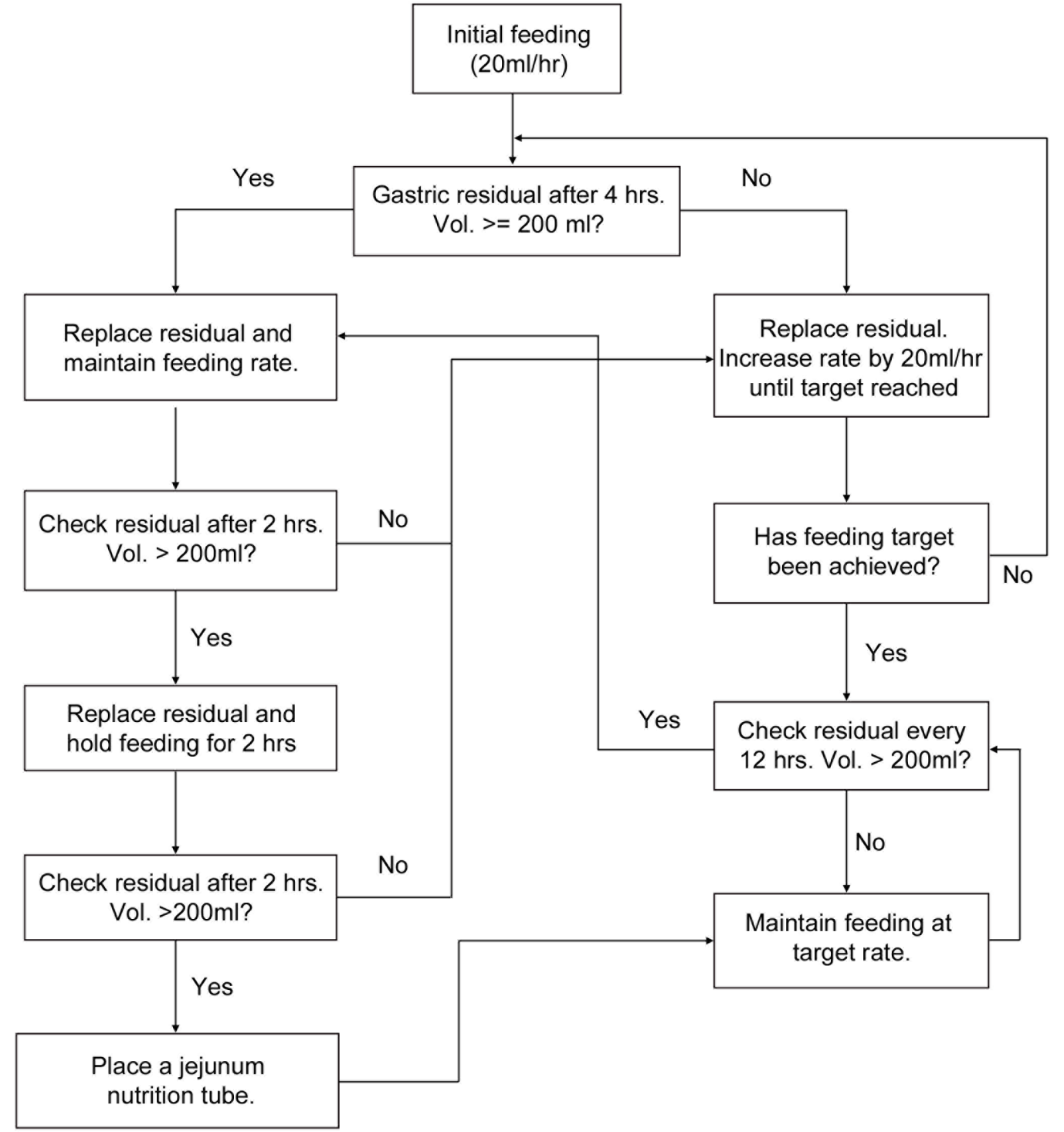

Figure 1 Enteral nutrition protocol for all included patients.

\section{Intervention}

All researchers are ICU clinicians and have received training for the EN protocol before this trial. EN support will be initiated within 24 hours after ICU admission and we use a simplistic ideal body weight-based equation to determine energy targets. ${ }^{16}$ The EN targets are $10 \mathrm{kcal} /$ $\mathrm{kg}$ ideal body weight/day and $25 \mathrm{kcal} / \mathrm{kg}$ ideal body weight/day of non-protein energy for the low-energy and high-energy groups, respectively. The protein $(1.4 \mathrm{~g} / \mathrm{kg}$ ideal body weight/day) will be given equally to these two groups. The intervention will last for 7 days and regular EN support will be applied to all patients once the 7-day intervention is completed. Successful intervention is defined as patients receiving at least $75 \%$ of EN target during the intervention period.

\section{EN protocol}

$\mathrm{EN}$ will be initiated at a rate of $20 \mathrm{~mL} /$ hour and gastric residual will be checked every 4 hours. The feeding rate will be increased by $20 \mathrm{~mL} /$ hour until the target feeding rate is reached if the residual volume is $<200 \mathrm{~mL}$. Otherwise, EN will be held or a jejunum nutrition tube will be placed. Once the feeding target is achieved, gastric residuals will be checked every 12 hours. Details of the enteral nutrition protocol are presented in figure 1.

\section{Outcome measures}

The primary outcome is all-cause mortality, Modified Rankin Scale and Glasgow Outcome Scale (GOS) at 90 days after enrolment. Secondary outcomes include hospital length of stay, ventilation duration, National Institutes of Health Stroke Scale (NIHSS), intra-abdominal pressure, EN-related complications and whole nosocomial infection rate. New nosocomial infections (blood, lungs, urinary tract or wounds, etc) are defined as any infection occurring within 48 hours after ICU admission. Aspiration pneumonia is one common EN-related complication. Yet, no established clinical symptoms are available to make a precise clinical distinction between pneumonia and aspiration pneumonia. Therefore, all episodes will be 
recorded as pneumonia, which is diagnosed according to the presence of respiratory system findings (cough, fever, tachypnoea and pathological respiratory sounds), blood tests and chest X-ray/thoracic CT presentations (newly developed infiltration). Moreover, the following variables will also be recorded at the time of enrolment as baseline characteristics: gender, age, weight, comorbidities, GCS, white blood cell count, plasma creatinine, plasma C-reactive protein, plasma albumin and pre-albumin level, lactate, fluid intake and urinary output, APACHE II, and sequential organ failure assessment (SOFA) scores.

\section{Data analyses}

Data analysis will be performed using the software STATA 12.0 (College Station, TX, USA). A blinded statistician will perform all analyses, based on the intention-to-treat principle for patients who have been assigned. The result using the per-protocol method will also be reported for patients who complete the whole trial. Variables will be reported using means (and SD), medians (and IQR) or proportions as appropriate, and appropriate statistical methods will be applied. All tests will be two-sided, and a significance level of $5 \%$ adopted.

\section{Ethics and dissemination}

The protocol has been approved by the ethics committee of Dongyang People's Hospital and has been registered at the Chinese Clinical Trial Registry (ChiCTR-INR-17011384). The trial will help to demonstrate whether or not low-energy EN is better than high-energy support in TICH patients. The results of this study will be published in peer reviewed journals for dissemination.

\section{DISCUSSION}

To the best of our knowledge, this is the first study protocol of a randomised, single-blind, controlled trial focusing on the appropriate amount of EN for TICH patients. In order to evaluate the crude efficacy of low-energy EN and calculate appropriate sample size, we performed a preliminary trial including 70 patients before this study and discovered a decreasing but insignificant trend of mortality in the low-energy group $(3 / 40$ vs $5 / 30, \mathrm{P}=0.233)$. We hope this trial will provide evidence regarding an appropriate EN strategy for TICH patients.

A high-quality methodology is essential for high validity and reliability of randomised trials. The limitation of this study is obvious as the clinicians are unlikely to be blinded in this trial. Yet, randomised open-label methodology has been used in several profound stroke trials. ${ }^{17} 18$ To alleviate potential bias caused by clinician unblinding, the outcome assessor will be unaware of allocation and will remain blinded until the whole trial has been completed. This is extremely important as all the neurological outcomes can be easily affected by the subjective opinion of the assessor.

Another anticipated limitation is that the volume of EN will sometimes be compromised, especially in the high-energy EN group as it will be easily affected by various factors such as delayed gastric emptying, ${ }^{8}$ diarrhoea and abdominal distension. In order to avoid EN overlap with the low-energy group, complete intervention is defined as patients receiving $>75 \%(25 \mathrm{kcal} / \mathrm{kg} /$ day $*$ $75 \%=18.75 \mathrm{kcal} / \mathrm{kg} /$ day) of EN target.

A third limitation is that this trial is being performed in only one teaching hospital. If this trial suggests a significant benefit, larger multicentre randomised trials will be needed in order to reach the goal of exploring appropriate EN strategies for patients with ICH.

Notwithstanding these limitations, this trial is well designed and we hope the results will provide new evidence about EN support for TICH patients.

Contributors YS: study design and manuscript writing. WZ: performing the preliminary study and collecting data. XC: data collection and analysis. MY: critical revision and approval of the manuscript. XJ: participated in the study design. KD: critical revision and submission.

Competing interests None declared.

Patient consent Obtained.

Ethics approval Ethics committee board of Dongyang people's hospital.

Provenance and peer review Not commissioned; externally peer reviewed.

Open Access This is an Open Access article distributed in accordance with the Creative Commons Attribution Non Commercial (CC BY-NC 4.0) license, which permits others to distribute, remix, adapt, build upon this work non-commercially, and license their derivative works on different terms, provided the original work is properly cited and the use is non-commercial. See: http://creativecommons.org/ licenses/by-nc/4.0/

(c) Article author(s) (or their employer(s) unless otherwise stated in the text of the article) 2017. All rights reserved. No commercial use is permitted unless otherwise expressly granted.

\section{REFERENCES}

1. Peeters W, van den Brande R, Polinder S, et al. Epidemiology of traumatic brain injury in Europe. Acta Neurochir 2015;157:1683-96.

2. Barlow KM. Traumatic brain injury. Handb Clin Neurol 2013;112:891-904.

3. Young B, Ott L, Yingling B, et al. Nutrition and brain injury. J Neurotrauma 1992;9:S375-83.

4. Jolliet P, Pichard C, Biolo G, et al. Enteral nutrition in intensive care patients: a practical approach. Clin Nutr 1999;18:47-56.

5. Seule M, Brunner T, Mack A, et al. Neurosurgical and intensive care management of traumatic brain injury. Facial Plast Surg 2015;31:325-31.

6. Taylor SJ, Fettes SB, Jewkes C, et al. Prospective, randomized, controlled trial to determine the effect of early enhanced enteral nutrition on clinical outcome in mechanically ventilated patients suffering head injury. Crit Care Med 1999;27:2525-31.

7. Grahm TW, Zadrozny DB, Harrington T. The benefits of early jejunal hyperalimentation in the head-injured patient. Neurosurgery 1989;25:729-35.

8. Ott L, Young B, Phillips R, et al. Altered gastric emptying in the head-injured patient: relationship to feeding intolerance. $J$ Neurosurg 1991;74:738-42.

9. Saxe JM, Ledgerwood AM, Lucas CE, et al. Lower esophageal sphincter dysfunction precludes safe gastric feeding after head injury. J Trauma 1994;37:581-6.

10. Arabi YM, Tamim HM, Dhar GS, et al. Permissive underfeeding and intensive insulin therapy in critically ill patients: a randomized controlled trial. Am J Clin Nutr 2011;93:569-77.

11. Casaer MP, Wilmer A, Hermans G, et al. Role of disease and macronutrient dose in the randomized controlled EPaNIC trial: a post hoc analysis. Am J Respir Crit Care Med 2013;187:247-55.

12. Arabi YM, Haddad SH, Tamim HM, et al. Near-target caloric intake in critically ill medical-surgical patients is associated with adverse outcomes. JPEN J Parenter Enteral Nutr 2010;34:280-8. 
13. Petros $\mathrm{S}$, Horbach M, Seidel F, et al. Hypocaloric vs normocaloric nutrition in critically ill patients: a prospective randomized pilot trial. JPEN J Parenter Enteral Nutr 2016;40:242-9.

14. McClave SA, Martindale RG, Vanek VW, et al. Guidelines for the provision and assessment of nutrition support therapy in the adult critically III patient: Society of Critical Care Medicine (SCCM) and American Society for Parenteral and Enteral Nutrition (ASPEN). JPEN J Parenter Enteral Nutr 2009;33:277-316.

15. Rice TW, Mogan S, Hays MA, et al. Randomized trial of initial trophic versus full-energy enteral nutrition in mechanically ventilated patients with acute respiratory failure. Crit Care Med 2011;39:967-74.
16. Taylor BE, McClave SA, Martindale RG, et al. Guidelines for the provision and assessment of nutrition support therapy in the adult critically ill patient: Society of Critical Care Medicine (SCCM) and American Society for Parenteral and Enteral Nutrition (ASPEN). Crit Care Med 2016;44:390-438.

17. Campbell BC, Mitchell PJ, Kleinig TJ, et al. Endovascular therapy for ischemic stroke with perfusion-imaging selection. N Engl J Med 2015;372:1009-18.

18. Goyal M, Demchuk AM, Menon BK, et al. Randomized assessment of rapid endovascular treatment of ischemic stroke. $N$ Engl $J$ Med 2015;372:1019-30. 